\title{
LA PRÉVALENCE DES HELMINTHOSES BOVINES A-T-ELLE ÉTÉ MODIFIÉE PAR LA CANICULE DE L'ÉTÉ 2003 dANS LE SUD-OUEST DE LA FRANCE ?
}

\author{
THOMAS C.*, JACQUIET P.* \& DORCHIES P.*
}

Summary: DOES 2003 SUMMER HEAT WAVE MODIFIED PREVALENCE OF BOVINE HELMINTHOSIS IN SOUTHWESTERN FRANCE?

The heat wave of 2003 summer had serious consequences on helminths prevalence and epidemiology. Comparison of 1,917 cattle epg counts in Southwestern France for years before and after showed that prevalence and mean numbers of Fasciola hepatica, paramphistomids, Dicrocœlium lanceolatum and gastrointestinal strongyles eggs were significantly reduced. Nevertheless, the next year the epg counts were quickly increasing. Several factors may have induced a more or less long modification: strains of helminths may become adapted, a new spreading of intermediate hosts may occur with a modified ecology and finally moving of hosts and reservoirs may be amongst causes of epidemiological changes. Finally, after a heat wave the anthelmintic pressure may be maintained, as parasites seem quickly become adapted to a novel and modified environment.

KEY WORDS : cattle, strongyles, Fasciola hepatica, paramphistomids, Dicrocoelium lanceolatum, heat wave.

\section{INTRODUCTION}

$\tau$ a température et l'humidité conditionnent l'évolution et la survie des œufs et des larves d'helminthes dans les pâturages. Lors d'accidents climatiques, on constate plus ou moins rapidement des conséquences sur la prévalence des infestations et sur leur traduction pathologique. Si les hivers doux et humides étaient connus comme favorables aux épisodes de fasciolose aiguë, les effets des températures estivales très élevées, associées à une sécheresse prolongée, n'ont jamais été véritablement évalués dans les pays tempérés bien que des synthèses (Afssa, 2005) et des observations ponctuelles aient été publiées récemment (Pritchard et al., 2005; Eysker et al., 2005). Au cours de l'année 2003, caractérisée par une canicule historique, tous les indicateurs montraient une baisse considérable de la pression parasitaire accompagnée d'une diminution significative des interventions vétérinaires. L'année suivante, en 2004, une baisse apparente du parasitisme

\footnotetext{
* Laboratoire de parasitologie, École nationale vétérinaire, 23, Chemin des Capelles, 31076 Toulouse Cedex, France.

Correspondance : Pr Philippe Dorchies.

Tél. : 33 (0) 561193871 - Fax : 33 (0) 561193944.

E-mail : p.dorchies@envt.fr
}

\section{Résumé :}

La comparaison des résultats de 1917 examens coproscopiques de bovins du midi toulousain montre qu'entre 2002 et 2003, la prévalence et le nombre moyen d'oeufs de grande douve, de paramphistomes, de petite douve et de strongles gastro-intestinaux ont significativement diminué. En revanche, dès l'année suivante, les niveaux parasitaires ont été restaurés. La discussion envisage les différents facteurs qui, à plus ou moins long terme, pourraient aboutir à une évolution durable de l'épidémiologie des helminthoses. Il s'agit d'une adaptation des souches de vers, d'une modification de l'écologie des hôtes intermédiaires et enfin d'un déplacement des hôtes ou des réservoirs. En bilan, un accident climatique comme celui de 2003 ne doit pas aboutir à un relâchement des mesures de lutte contre le parasitisme mais à leur adaptation en étudiant un nouveau calendrier des interventions.

MOTS CLÉS : bovins, Strongles, Fasciola hepatica, paramphistomidés, Dicrocœlium lanceolatum, canicule.

était constatée. L'année de canicule de 2003 avait-t-elle eu un effet sur les helminthes, en particulier sur ceux dont le cycle nécessite le passage par un hôte intermédiaire aquatique comme la grande douve et les paramphistomes?

Nous recevons régulièrement au laboratoire de parasitologie de l'École nationale vétérinaire de Toulouse, des prélèvements en vue d'examens coproscopiques. Nous avons pensé que la comparaison des résultats obtenus en 2002 avant la grande canicule avec ceux de 2003 et de 2004 pouvait peut-être donner des indications intéressantes sur l'évolution à court terme du parasitisme et sur les conséquences à en tirer pour organiser la lutte

\section{MATÉRIEL ET MÉTHODES}

T es échantillons de matières fécales provenaient de la clinique de pathologie bovine de l'École nationale vétérinaire de Toulouse ainsi que des clientèles vétérinaires du midi toulousain recouvrant la vallée de la Garonne, les départements limitrophes du Gers et du Tarn ainsi que le piémont pyrénéen de l'Ariège. Le climat est de type océanique atténué, compte tenu de la proximité de la frontière climatique 
avec le climat méditerranéen située au seuil de Naurouze à moins de $80 \mathrm{~km}$ de Toulouse. Les bovins élevés dans la région sont des Prim'Holstein, des Limousines, des Gasconnes et des Blondes d'Aquitaine.

Les examens de routine ont été pratiqués en lame de Mac Master utilisant l'iodomercurate de potassium comme solution dense (densité 1,44). La lecture a été faite sur lame totale avec une flottation en tube permettant une sensibilité à sept oufs par gramme de matières fécales. Au total 1917 examens individuels ont été réalisés : 792 en 2002, 537 en 2003 et 588 en 2004.

Analyse statistique : un test du $\mathrm{Chi}^{2}$ a été réalisé à partir des pourcentages comparés deux à deux suivant la nature du parasite et l'année.

\section{RÉSULTATS}

I e tableau I récapitule les résultats concernant les quatre helminthes les plus fréquemment rencontrés dans la région. Les œufs de Nematodirus, de trichures et de cestodes, ainsi que les coccidies ne sont pas pris en compte en raison du petit nombre de résultats positifs.

Tous les tests réalisés sur les prévalences annuelles moyennes sont significatifs, sauf pour la grande douve entre 2003 et 2004 et pour la petite douve entre 2002 et 2003 .

Les figures 1 et 2 rassemblent les résultats en quatre périodes qui correspondent à des moments particuliers du cycle d'élevage : janvier à mars, durant lesquels les animaux sont en stabulation, avril à juin, puis juillet à septembre et enfin octobre à décembre.

\begin{tabular}{|c|c|c|}
\hline $\begin{array}{l}2002: 792 \text { examens } \\
2003: 537 \text { examens } \\
2004: 588 \text { examens }\end{array}$ & $\begin{array}{c}\% \\
\text { d'examens } \\
\text { positifs }\end{array}$ & $\begin{array}{c}\text { Nombre moyen } \\
\text { d'ouufs/gramme } \\
\text { de matières } \\
\text { fécales }\end{array}$ \\
\hline \multicolumn{3}{|l|}{ Grande douve } \\
\hline 2002 & $4,9 \%$ & 31,8 \\
\hline 2003 & $2,4 \%$ & 7 \\
\hline 2004 & $1,4 \% 0^{\mathrm{b}}$ & 37,4 \\
\hline \multicolumn{3}{|l|}{ Paramphistomes } \\
\hline 2002 & $49,4 \%{ }^{\mathrm{a}}$ & 139,4 \\
\hline 2003 & $25,7 \%{ }^{\mathrm{b}}$ & 57,7 \\
\hline 2004 & $31,8 \%{ }^{\mathrm{c}}$ & 87,9 \\
\hline \multicolumn{3}{|l|}{ Petite douve } \\
\hline 2002 & $18,43 \%{ }^{\mathrm{a}}$ & 36,3 \\
\hline 2003 & $16,4 \%{ }^{\mathrm{a}}$ & 14,4 \\
\hline 2004 & $26,7 \%{ }^{\mathrm{b}}$ & 23,8 \\
\hline \multicolumn{3}{|l|}{ Strongles digestifs } \\
\hline 2002 & $48 \%{ }^{\mathrm{a}}$ & 109 \\
\hline 2003 & $42,3 \%{ }^{\mathrm{b}}$ & 59,7 \\
\hline 2004 & $79,4 \%{ }^{c}$ & 56,3 \\
\hline
\end{tabular}

Tableau I. - Résultats des examens coproscopiques*.

* Pour chaque parasite, les valeurs suivies de lettres différentes sont significativement différentes.

\section{DISCUSSION}

I a conséquence la plus apparente de la canicule est la chute du nombre de bovins éliminant des œeufs de grande douve. En revanche, les animaux rejetant des oufs de paramphistomes, de petites douves ou de strongles sont statistiquement plus nombreux en 2004. Ceci montre qu'après l'accident climatique de 2003, la diminution du parasitisme n'est que transitoire

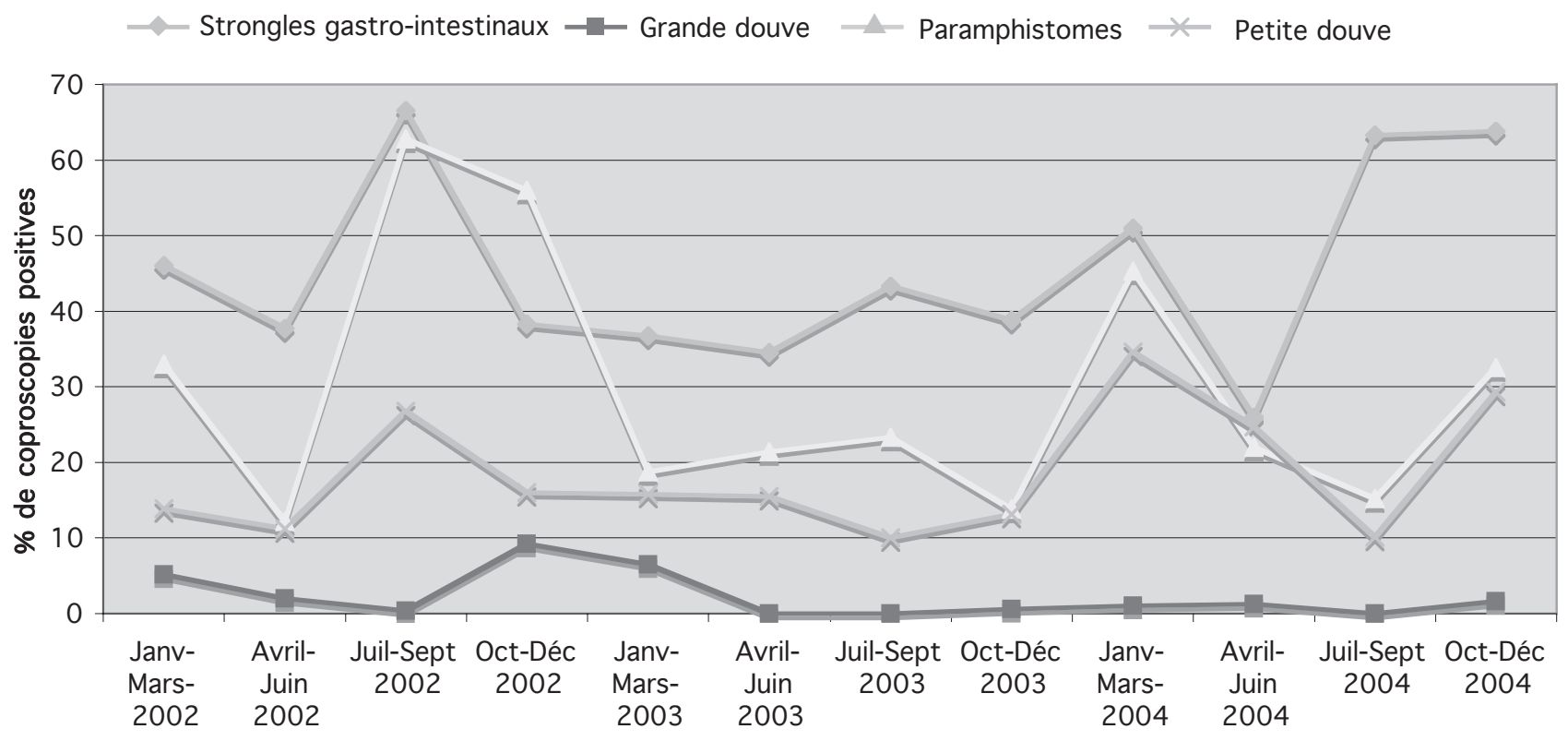

Fig. 1. - Évolution des prévalences saisonnières des helminthoses. 


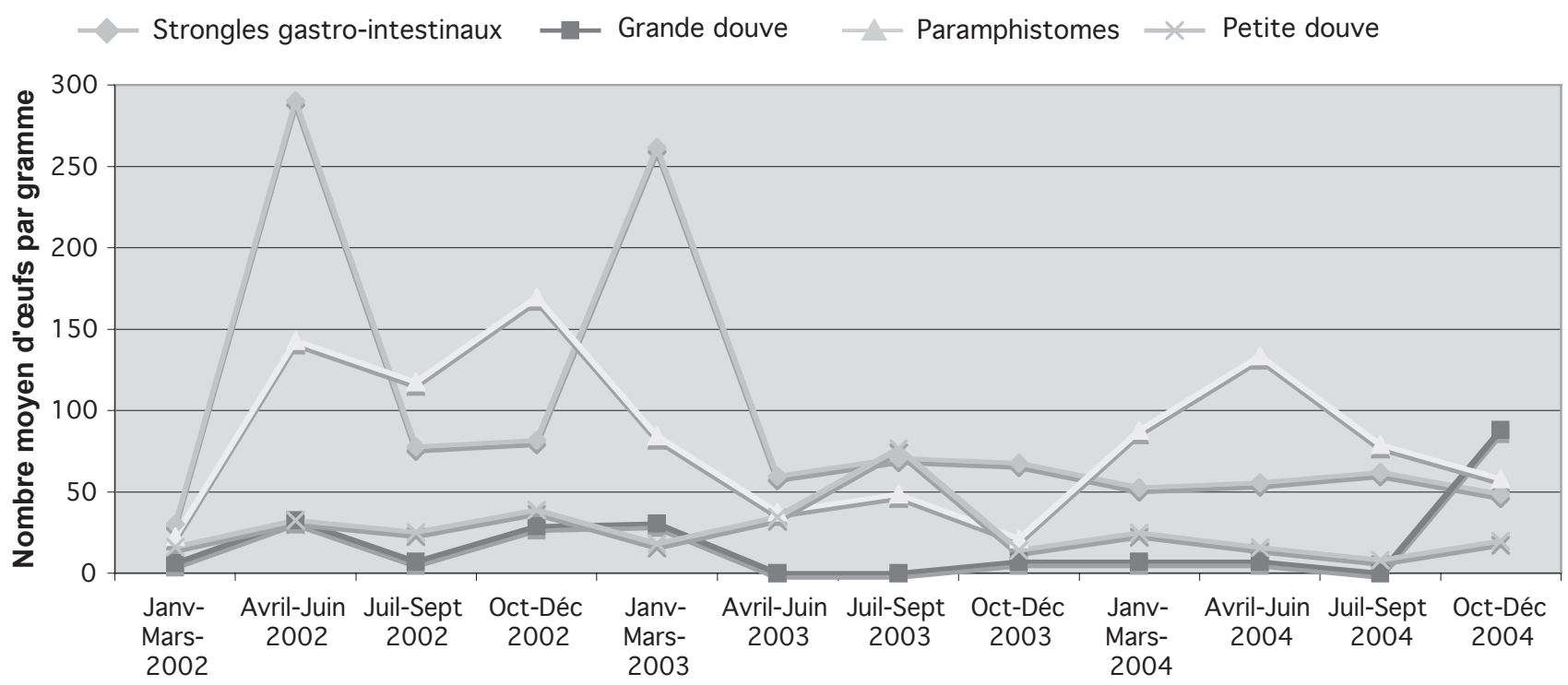

Fig. 2. - Évolution du nombre moyen d'œufs par gramme de matières fécales.

et que les capacités d'adaptation des helminthes suppléent rapidement les conséquences de la sécheresse. Les conditions météorologiques interviennent directement sur la survie des œufs et des larves mais surtout sur la cinétique de leur évolution. L'humidité et l'oxygénation sont des facteurs statiques indispensables, mais la température est le facteur dynamique apportant l'énergie nécessaire à l'évolution. Celle-ci n'est possible que dans une certaine fourchette de températures optimales, les extrêmes, comme ceux qui ont été subis en 2003, sont létaux pour les formes libres. Ainsi, la canicule décontamine les pâturages en détruisant les formes libres, mais les œufs ou les parthenita hébergés par les hôtes intermédiaires sont protégés. Par ailleurs, les strongles gastro-intestinaux se rencontrent sous des climats variés, et, dans les zones les plus arides, leur survie est aussi assurée par la persistance d'adultes ou de larves en hypobiose. L'observation faite par Eysker et al. (2005) montre que dans les conditions européennes ce type d'adaptation est ponctuellement possible : l'allongement de la durée de vie des adultes d'Hamonchus contortus a assuré la pérennité de l'infestation des moutons.

Les changements climatiques peuvent aussi avoir une influence sur la présence, la survie et la pullulation des hôtes intermédiaires : mollusques (aquatiques ou xérothermiques) et fourmis. Pour Fasciola hepatica, nos résultats montrent une diminution de la prévalence et de l'intensité de la ponte des vers. Les limnées sont très résistantes à la sécheresse, ayant la possibilité de s'enfouir plus ou moins profondément dans le sol, assurant ainsi la survie des larves de trématodes qu'elles hébergent, en revanche, leurs pontes sont détruites par la chaleur, la sécheresse, le froid et le gel. Pour ces mollusques, la conséquence la plus importante d'un réchauffement climatique avec un adoucissement des hivers serait un allongement de leur période d'activité et de reproduction. Concomitamment, l'éclosion des miracidiums se produisant durant toute la période où la moyenne dépasse $10^{\circ} \mathrm{C}$, l'infestation des limnées serait possible plus longtemps en automne, en hiver et au printemps : la fasciolose et la paramphistomose seraient les grandes bénéficiaires du réchauffement climatique si les pluies d'hiver se maintenaient.

Il faut enfin tenir compte de ce que la répétition des traitements douvicides provoque une diminution globale des charges parasitaires chez les bovins. Les coproscopies sont à des niveaux faibles et ce n'est que par l'augmentation de la quantité de matières fécales examinées que l'on peut dépister des infestations pauciparasitaires infra cliniques (Rapsch et al., 2006). Ainsi, lors d'une enquête sur 310 bovins, l'examen de 5 grammes de matières fécales n'a révélé que 35 animaux (11,3\%) éliminant des oufs de grande douve alors que l'examen de 50 grammes avec une méthode d'enrichissement par tamisage a permis d'en trouver 95 (31,6\%) (Salem et al., 2007). En conséquence de ces faibles quantités d'œufs évacués, on assiste à une diminution notable du nombre et de la densité des miracidiums : Dreyfuss et al. (1999) ont montré que les pauci-infestations des limnées étaient plus favorables à leur survie et à l'évolution ultérieure des parthenita que les infestations plus importantes. Sur le plan épidémiologique, ces observations sont capitales : la production des métacercaires est plus élevée chez les limnées infectées par un seul miracidium. Les dommages subis par les mollusques du fait de la sécheresse se trouvent ainsi annulés par l'augmentation de la fertilité des formes larvaires chez la limnée. En bilan, la chaleur et la sécheresse, à court terme, ne modifient que peu la population de grande douve. 
Pour les paramphistomes, les conditions sont encore plus favorables car plusieurs espèces de limnées et de planorbes interviennent comme hôtes intermédiaires. En ce qui concerne la petite douve, on sait que les températures comprises entre 7,5 et $26,9^{\circ} \mathrm{C}$ favorisent les infestations puisque les fourmis vont rester fixées par tétanisation aux herbes. Les périodes de canicule n'interviennent pas puisque pendant l'été les infestations ne se produisent pas (Manga-Gonzalez \& GonzalezLanza, 2005). Comme pour la grande douve c'est l'atténuation des conditions hivernales qui assure une augmentation des risques d'infestation par la survie et l'activité prolongées des hôtes intermédiaires, et donc des formes larvaires qui seront plus nombreuses.

Enfin, la canicule de 2003 n'a pas entraîné fondamentalement de modifications de la répartition des animaux. Il faut cependant noter que les bas-fonds et les points d'eau ont subi un surpeuplement temporaire aboutissant à une contamination plus élevée du sol, celui-ci, plus riche en matières organiques a été ensuite plus favorable à l'évolution des larves infestantes et à la pullulation des limnées. De même, les hôtes sauvages comme les ragondins ont pu continuer à pulluler en hiver; la forte prévalence de leur infestation par la grande douve ne peut encore qu'augmenter et en parallèle les facteurs de risque pour les bovins.

\section{CONCLUSION}

Tace à des températures extrêmes et à une séche$\checkmark$ resse importante, aucune modification durable du 1 polyparasitisme n'apparaît chez les bovins : cela montre, une fois encore, la grande aptitude adaptative des parasites. Les diverses stratégies de survie compensent les pertes importantes, mais les charges parasitaires plus faibles hébergées par les animaux peuvent présenter à terme un danger de persistance de sources de parasites méconnues car difficile à dépister et ne se traduisant par aucune conséquence pathologique et donc économique notable. Il est donc nécessaire d'être particulièrement vigilant et de ne pas assimiler sécheresse à diminution globale et durable des helminthoses.

\section{RÉFÉRENCES}

Afssa. Rapport sur l'évaluation du risque d'apparition et de développement de maladies animales compte tenu d'un éventuel réchauffement climatique, 2005. http://afssa.fr

Dreyfuss G., Vignoles P., Rondelaud D. \& Vareille-Morel C. Fasciola hepatica: characteristics of infection in Lymnaea truncatula in relation to the number of miracidia at exposure. Experimental Parasitology, 1999, 92, 19-23.

Eysker M., Bakker N., Kooyman F.N.J., Van der Linden D., Schrama C. \& Ploeger H.W. Consequences of the unusually warm and dry summer of 2003 in the Netherlands: poor development of free living stages, normal survival of infective larvæ and long survival of adult gastrointestinal nematodes of sheep. Veterinary Parasitology, 2005, 133, 313-321.

Manga-Gonzalez M.Y. \& GonZalez-Lanza C. Field and experimental studies on Dicrocoelium dendriticum and dicrocoeliais in northern Spain. Journal of Helminthology, 2005, 79, 291-302.

Pritchard G.C., Forbes A.B., Williams D.J.L., Salimi-Bejestami M.R. \& Daniel R.G. Emergence of fasciolosis in cattle in East Anglia. Veterinary Record, 2005, 157, 578-582.

Rapsch C., Schweizer G., Grimm F., Kohler L., Bauer C., Deplazes P., Braun U. \& Torgerson P.R. Estimating the true prevalence of Fasciola hepatica in cattle slaughtered in the absence of an absolute diagnostic test. International Journal for Parasitology, 2006, 36, 1153-1158.

SAlem A., Jacquiet P., Chauvin A. \& Dorchies P. Estimating the prevalence of Fasciola bepatica in cattle: which method is the best diagnostic test? WAAVP Congress, Ghent, 2007.

Reçu le 26 juin 2007 Accepté le 4 juillet 2007 